\title{
Ensayo controlado citisina versus placebo para la cesación del tabaquismo
}

\author{
Placebo-controlled trial of cytisine for smoking \\ cessation
}

Claudia Zuluaga Serna, MD.(1), Alejandra Cañas Arboleda, MD. ${ }^{(2)}$

(1)Residente primer año Medicina Interna, Pontificia Universidad Javeriana, Hospital Universitario San Ignacio, Bogotá, Colombia. (2)Internista, Neumóloga, Hospital Universitario San Ignacio, Profesora Asociada Pontificia Universidad Javeriana, Bogotá, Colombia. Correspondencia: Alejandra Cañas Arboleda, correo electrónico: alejandra.canas@javeriana. edu.co

Recibido: 06/08/14. Aceptado: 10/09/14.

\section{Referencia}

West R, Zatonski W, Cedzynska M, Lewandowska D, Pazik J, Aveyard $\mathrm{P}$, et al. Placebo-controled trial of cysticine for smoking cessation. Engl J Med. 2011; 365(13):1193-00.

\section{Pregunta}

En pacientes fumadores ¿Es la terapia con citisina mejor que el placebo para ayudar a dejar de fumar?

\section{Diseño}

Estudio de asignación aleatoria, doble ciego, controlado con placebo con un grupo paralelo.

\section{Cegamiento}

Doble ciego.

\section{Periodo de seguimiento}

El reclutamiento inició el 10 de diciembre de 2007. Todos los pacientes se siguieron durante doce meses y el último reclutamiento se hizo el 2 de septiembre de 2010.

\section{Lugar}

Centro Maria Sklodowska-Curie Memorial Cancer Center, Warsaw, Polonia.

\section{Pacientes}

Pacientes que fumaban diez o más cigarrillos al día y deseaban dejar de fumar. Se excluyeron embarazadas, en lactancia o que estuvieran planeando un embarazo, con hipertensión arterial no controlada, enfermedad aterosclerótica severa diagnosticada o diagnóstico de desorden psiquiátrico. No se incluyeron fumadores que tuvieran enfermedad avanzada relacionada con el cigarrillo.

\section{Intervención}

Se aleatorizaron 740 pacientes en dos grupos de 370 cada uno; uno recibió citisina y el otro placebo. Todos los pacientes recibieron terapia comportamental y asistían a un número mínimo de sesiones de seguimiento para mantener el estímulo para la cesación. La visita inicial fue de aleatorización y entrega del medicamento. Se le suministraba un régimen de tabletas durante 25 días, con tabletas de $1,5 \mathrm{mg}$; esquema: una tableta cada 2 horas (día 1 a 3), 5 tabletas al día (día 4 al 9), 4 tabletas por día (día 13 a 16), 3 tabletas al día (día 17 al 20) y 2 tabletas al día (día 21 a 25). Posteriormente recibían una llamada 
Tabla 1. Efecto de la citisina en la cesación del tabaco *

\begin{tabular}{|c|c|c|c|c|}
\hline \multirow[t]{2}{*}{ Desenlaces } & $\begin{array}{l}\text { Citisina } \\
(n=370)\end{array}$ & $\begin{array}{r}\text { Placebo } \\
(n=370)\end{array}$ & \multirow{2}{*}{$\begin{array}{l}\text { Diferencia } \\
\text { en puntos de } \\
\text { porcentaje } \\
\text { (IC95\%) }\end{array}$} & \multirow[t]{2}{*}{$\begin{array}{c}\text { Tasa relativa } \\
\text { (IC95\%) }^{\dagger}\end{array}$} \\
\hline & \multicolumn{2}{|c|}{ Porcentaje (n) } & & \\
\hline $\begin{array}{l}\text { Desenlace primario: abstinencia a } \\
12 \text { meses }\end{array}$ & $8,4(31)$ & $2,4(9)$ & $6,0(2,7-9,2)^{\ddagger}$ & $3,4(1,7-7,1)$ \\
\hline Abstinencia a 6 meses & $10,0(37)$ & $3,5(13)$ & $6,5(2,9-10,1)^{\ddagger}$ & $2,9(1,5-5,3)$ \\
\hline Punto de prevalencia a 12 meses & $13,2(49)$ & $7,3(27)$ & $5,9(1,6-10,3)^{1}$ & $1,8(1,2-2,8)$ \\
\hline
\end{tabular}

*El desenlace primario fue abstinencia a 12 meses después de finalizar el tratamiento, de acuerdo con los criterios estándar de Russell. El desenlace secundario fue abstinencia a 6 meses de acuerdo con los criterios estándar de Russell. El punto de prevalencia a 12 meses se define como abstinencia en la semana previa a la visita de seguimiento. Los criterios Russell estándar incluyen la clasificación de pérdida hasta el seguimiento, así como falla en el tratamiento, verificación bioquímica de la abstinencia al final del seguimiento, y el autorreporte de continua abstinencia (menos de 5 cigarrillos fumados en cada uno de los 6 meses de seguimiento). No hubo asociaciones significativas entre las medidas basales y el desenlace primario.

†La tasa relativa es el porcentaje en el grupo de citisina dividido por el porcentaje del grupo placebo.

$\neq \mathrm{p}<0,001$.

$q p=0,01$.

Tabla 2. Eventos adversos reportados por diez o más participantes en el estudio*

\begin{tabular}{|lccrc|}
\hline \multicolumn{1}{|c}{ Evento } & $\begin{array}{c}\text { Citisina } \\
\mathbf{( n = 3 7 0 )}\end{array}$ & $\begin{array}{c}\text { Placebo } \\
\mathbf{( n = 3 7 0 )}\end{array}$ & $\begin{array}{c}\text { Punto de } \\
\text { diferencia de } \\
\text { porcentajes } \\
\text { (IC95\%) }\end{array}$ & $\begin{array}{c}\text { Tasa relativa } \\
\text { (IC95\%) }\end{array}$ \\
Cualquier evento gastrointestinal & Porcentaje (número de pacientes) & $5,7(1,2-10,2)^{\natural}$ & $1,7(1,6-2,6)$ \\
Epigastralgia & $13,8(51)$ & $8,1(30)$ & $0,8(-1,8-3,4)$ & $1,3(0,6-2,8)$ \\
Náuseas & $3,8(14)$ & $3,0(11)$ & $1,1(-1,5-3,6)$ & $1,4(0,6-3,1)$ \\
Dispepsia & $3,8(14)$ & $2,7(10)$ & $1,1 /-1,5-3,6)$ & $1,4(0,6-3,1)$ \\
Boca seca & $2,4(9)$ & $2,7(10)$ & $1,6(0-3,3)$ & $4(0,9-18,7)$ \\
Cualquier evento psiquiátrico & $2,2(8)$ & $0,5(2)$ & $1,4(-1,4-4,2)$ & $1,4(0,7-2,9)$ \\
Mareo & $4,6(17)$ & $3,2(12)$ & $1,1(-0,7-2,9)$ & $2,0(0,6-6,6)$ \\
Somnolencia & $2,2(8)$ & $1,1(4)$ & $0,5(-1,1-2,2)$ & $1,5(0,4-5,3)$ \\
Cualquier evento en el sistema & $1,6(6)$ & $1,1(4)$ & $0,3(-2,0-2,6)$ & $1,1(0,5-2,7)$ \\
nervioso & $2,7(10)$ & $2,4(9)$ & & \\
Cefalea & $1,9(7)$ & $2,2(8)$ & $-0,3(-2,3-1,8)$ & $0,9(0,3-2,4)$ \\
Piel y tejido celular subcutáneo & $1,6(6)$ & $1,4(5)$ & $0,3(-1,5-2,0)$ & $1,2(0,4-3,9)$ \\
\hline
\end{tabular}

*La incidencia de eventos se analizó de acuerdo con la categorización y términos preferidos por el "Medical Dictionary for Regulatory Activities System Organ Class (SOC)". Los participantes que reportaron más de un evento en un sistema, fueron contabilizados sólo una vez en la misma categoría. Las categorías SOC para otros eventos (aquellos reportados por menos de 10 participantes) fueron las siguientes: generales ( 5 en citisina y 5 con placebo), cardiacos ( 4 citisina y 2 placebo), infecciones ( 1 con placebo), sistema inmune ( 1 con placebo) y metabolismo y nutrición (1 con placebo).

†Las diferencias se calcularon de acuerdo con valores no redondeados.

$\ddagger E l$ riesgo relativo es el porcentaje en el grupo citisina dividido por el porcentaje en el placebo.

$\{p=0,02$. No hubo otras diferencias significativas.

telefónica el día de la suspensión del tabaco, una semana después, y con una visita opcional. Luego recibían una visita clínica 4 semanas después del día de suspensión del tabaco y finalmente se hacían llamadas de seguimiento a los 6 y 12 meses a fin de averiguar por la abstinencia.

\section{Resultados}

El desenlace primario fue la tasa de abstinencia a 12 meses, la cual fue de 8,4\% (31 participantes) en el grupo de citisina comparado con $2,4 \%$ ( 9 participantes) en el placebo, con una diferencia del $6 \%$ 
de puntos (IC 85\% 2,3-9,2; p=0,001) (tabla 1). El punto de prevalencia de 7 días para abstinencia a 12 meses de seguimiento, fue de $13,2 \%$ en citisina versus $7,3 \%$ con placebo $(p=0,01)$.

El desenlace secundario fue la abstinencia sostenida por los primeros 6 meses y el punto de prevalencia a los 12 meses de seguimiento. La abstinencia sostenida a los 6 meses fue diez veces más probable en el grupo de citisina que en el placebo, donde fue sólo tres veces más probable y el resultado fue estadísticamente significativo, con una diferencia de 6,5 puntos por ciento (IC $2,9-10,1 ; p<0,001$ ). Las diferencias del efecto de la citisina vs. placebo fueron más bajas que las de la vareniclina y la terapia de reemplazo con nicotina en otros estudios.

En el grupo de citisina se reportaron con más frecuencia efectos adversos de tipo gastrointestinal, que en el grupo placebo (tabla 2).

\section{Conclusión}

La citisina fue efectiva para la cesación del tabaco en este ensayo. Su costo bajo comparado con otras farmacoterapias, la convierten en una opción y un tratamiento atractivo para fumadores en países de bajos o medianos ingresos. Estos resultados fueron en un único centro.

\section{Citisina comparado con nicotina para la cesación del tabaquismo}

\section{Referencia}

Walker N, Howe C, McRobbie H, Barnes J, Nosa V, Parag V, et al. Cistisine versus nicotine for smoking cessation. N Engl J Med. 2014;371(25):2353-62.

\section{Pregunta}

En pacientes fumadores ¿Es la terapia con citisina tan efectiva como la terapia de reemplazo con nicotina para ayudar a dejar de fumar?

\section{Diseño}

Estudio de no inferioridad con asignación aleatoria, abierto.

\section{Cegamiento}

Estudio abierto.

\section{Periodo de seguimiento}

6 meses.

\section{Lugar}

Se eligieron los pacientes que llamaron a la línea de abandono del tabaco de Nueva Zelanda.

\section{Pacientes}

Se incluyeron 1.310 pacientes mayores de 18 años, quienes fumaban a diario, con un grado de dependencia al cigarrillo intermedio calculado por la escala de Fagerstrom de 1 a 10 que se interpreta como alta dependencia por encima de 5 y baja si está por debajo de esta cifra.

\section{Intervención}

A todos los participantes se les ofreció vía telefónica terapia comportamental de baja intensidad (un promedio de 3 llamadas de 10 a 15 minutos en 8 semanas). Se asignaron de forma aleatoria a dos grupos: uno a terapia de reemplazo con nicotina por 8 semanas (dosis de acuerdo con la guía local para dejar de fumar y la preferencia del participante para decidir la presentación preferida de nicotina parches de $7 \mathrm{mg}, 14 \mathrm{mg}$ y $21 \mathrm{mg}$, chicles de $2 \mathrm{mg}, 4 \mathrm{mg}$ y tabletas masticables de $1 \mathrm{mg}$ y $2 \mathrm{mg}$ ) y otro que recibió terapia con citisina (curso de 25 días con suspensión del tabaco el quinto día de terapia (día 1-3: 6 tabletas al día, día 4-12: 5 tabletas al día, día 13-16: 4 tabletas a día, día 17-20: 3 tabletas al día y día 21-25: 2 tabletas al día). 
de puntos (IC 85\% 2,3-9,2; p=0,001) (tabla 1). El punto de prevalencia de 7 días para abstinencia a 12 meses de seguimiento, fue de $13,2 \%$ en citisina versus $7,3 \%$ con placebo $(p=0,01)$.

El desenlace secundario fue la abstinencia sostenida por los primeros 6 meses y el punto de prevalencia a los 12 meses de seguimiento. La abstinencia sostenida a los 6 meses fue diez veces más probable en el grupo de citisina que en el placebo, donde fue sólo tres veces más probable y el resultado fue estadísticamente significativo, con una diferencia de 6,5 puntos por ciento (IC $2,9-10,1 ; p<0,001$ ). Las diferencias del efecto de la citisina vs. placebo fueron más bajas que las de la vareniclina y la terapia de reemplazo con nicotina en otros estudios.

En el grupo de citisina se reportaron con más frecuencia efectos adversos de tipo gastrointestinal, que en el grupo placebo (tabla 2).

\section{Conclusión}

La citisina fue efectiva para la cesación del tabaco en este ensayo. Su costo bajo comparado con otras farmacoterapias, la convierten en una opción y un tratamiento atractivo para fumadores en países de bajos o medianos ingresos. Estos resultados fueron en un único centro.

\section{Citisina comparado con nicotina para la cesación del tabaquismo}

\section{Referencia}

Walker N, Howe C, McRobbie H, Barnes J, Nosa V, Parag V, et al. Cistisine versus nicotine for smoking cessation. N Engl J Med. 2014;371(25):2353-62.

\section{Pregunta}

En pacientes fumadores ¿Es la terapia con citisina tan efectiva como la terapia de reemplazo con nicotina para ayudar a dejar de fumar?

\section{Diseño}

Estudio de no inferioridad con asignación aleatoria, abierto.

\section{Cegamiento}

Estudio abierto.

\section{Periodo de seguimiento}

6 meses.

\section{Lugar}

Se eligieron los pacientes que llamaron a la línea de abandono del tabaco de Nueva Zelanda.

\section{Pacientes}

Se incluyeron 1.310 pacientes mayores de 18 años, quienes fumaban a diario, con un grado de dependencia al cigarrillo intermedio calculado por la escala de Fagerstrom de 1 a 10 que se interpreta como alta dependencia por encima de 5 y baja si está por debajo de esta cifra.

\section{Intervención}

A todos los participantes se les ofreció vía telefónica terapia comportamental de baja intensidad (un promedio de 3 llamadas de 10 a 15 minutos en 8 semanas). Se asignaron de forma aleatoria a dos grupos: uno a terapia de reemplazo con nicotina por 8 semanas (dosis de acuerdo con la guía local para dejar de fumar y la preferencia del participante para decidir la presentación preferida de nicotina parches de $7 \mathrm{mg}, 14 \mathrm{mg}$ y $21 \mathrm{mg}$, chicles de $2 \mathrm{mg}, 4 \mathrm{mg}$ y tabletas masticables de $1 \mathrm{mg}$ y $2 \mathrm{mg}$ ) y otro que recibió terapia con citisina (curso de 25 días con suspensión del tabaco el quinto día de terapia (día 1-3: 6 tabletas al día, día 4-12: 5 tabletas al día, día 13-16: 4 tabletas a día, día 17-20: 3 tabletas al día y día 21-25: 2 tabletas al día). 


\section{Resultados}

Entre 3.001 personas evaluadas, fueron elegibles 1.310 para ser aleatorizados en dos grupos de 655 pacientes a recibir citisina versus terapia de reemplazo con nicotina.

En el desenlace primario, citisina no fue inferior a la terapia de reemplazo con nicotina. Las tasas de abstinencia a 30 días fueron más altas en el grupo de citisina que en el de terapia de reemplazo con nicotina. El grupo citisina tuvo 9,3 veces más probabilidad de abandonar el tabaquismo que el de terapia con nicotina, con un número necesario a tratar de 11 pacientes para evidenciar el efecto. El análisis de subgrupo por sexos demostró más alta abstinencia con citisina a un mes en mujeres y no arrojó diferencia significativa (no inferioridad) en hombres (tabla 1).

El desenlace secundario de cesación a una semana, 1, 2 y 6 meses fue similar al desenlace primario con excepción en los 6 meses, cuando la diferencia no fue estadísticamente significativa.

Los eventos adversos reportados fueron mayores en el grupo de citisina que en el de terapia de reemplazo con nicotina; el primer grupo tuvo 1,7 veces más incidencia de efectos adversos que el segundo, con diferencia estadísticamente significativa (IC $1,4-2 ; \mathrm{p}>0,001)$.

\section{Conclusión}

La citisina fue superior a la terapia de reemplazo con nicotina como terapia para abandonar el tabaco entre los fumadores motivados a dejarlo. Los eventos adversos con la citisina fueron dos veces más comunes y similares a los observados en ensayos previos. Se evidenciaron más tasas de abandono en el subgrupo de mujeres, efecto que no había sido reportado previamente con agonistas parciales del receptor nicotínico $\alpha 4 \beta 2$.

Tabla 1. Desenlaces en los grupos de terapia combinada y placebo.

\begin{tabular}{|c|c|c|c|c|c|}
\hline & $\begin{array}{l}\text { Citisina } \\
(n=655)\end{array}$ & $\begin{array}{c}\text { TRN } \\
(n=655)\end{array}$ & $\begin{array}{l}\text { Riesgo relativo } \\
\text { (IC95\%) }\end{array}$ & $\begin{array}{l}\text { Diferencia de } \\
\text { riesgo } \\
\text { (IC95\%) }\end{array}$ & $\mathbf{P}$ \\
\hline & \multicolumn{2}{|c|}{ Número total/número \% } & & & \\
\hline \multicolumn{6}{|l|}{ Abstinencia continua } \\
\hline \multicolumn{6}{|l|}{ Tasa de abandono } \\
\hline 1 semana & $394(60)$ & $303(46)$ & $1,3(1,2-1,4)$ & $13,9(8,5-19,2)$ & $<0,001$ \\
\hline 1 mes & $264(40)$ & $203(31)$ & $1,3(1,1-1,5)$ & $9,3(4,2-14,5)$ & $<0,001$ \\
\hline 2 meses & $202(31)$ & $143(22)$ & $1,4(1,2-1,7)$ & $9,0(4,3-13,8)$ & $<0.001$ \\
\hline 6 meses & $143(22)$ & $100(15)$ & $1,4(1,1-1,8)$ & $6,6(2,4-10,8)$ & 0.002 \\
\hline \multicolumn{6}{|l|}{ Análisis de sensibilidad a 1 mes } \\
\hline Casos completos & $264 / 565(47)$ & $203 / 560(36)$ & $1,3(1,1-1,5)$ & $10,5(4,8-16,2)$ & $<0,001$ \\
\hline \multicolumn{6}{|l|}{ Por protocolo } \\
\hline $\begin{array}{l}\text { Población con violaciones al protocolo } \\
\text { excluyendo incumplimiento }\end{array}$ & $252 / 537$ & $100 / 551$ & $1,3(1,1-1,5)$ & $10,8(5,0-16,6)$ & $<0,001$ \\
\hline $\begin{array}{l}\text { Población con violaciones al protocolo } \\
\text { incluyendo incumplimiento }\end{array}$ & $189 / 330$ & $167 / 407$ & $1,4(1,2-1,6)$ & $16,2(9,1-23,4)$ & $<0,001$ \\
\hline \multicolumn{6}{|l|}{ Punto de prevalencia a siete días } \\
\hline \multicolumn{6}{|l|}{ Tasa de abandono } \\
\hline 1 semana & $266(41)$ & $199(30)$ & $1,3(1,2-1,6)$ & $10,2(5,1-15,4)$ & $<0,001$ \\
\hline 1 mes & $273(42)$ & $215(33)$ & $1,3(1,1-1,5)$ & $8,9(3,7-14,1)$ & $<0,001$ \\
\hline 2 meses & $246(38)$ & $206(32)$ & $1,2(1,0-1,4)$ & $6,1(1,0-11,3)$ & 0,020 \\
\hline 6 meses & 206 (31) & $196(30)$ & $1,1(0,9-1,2)$ & $1,5(-3,5-6,5)$ & 0,549 \\
\hline
\end{tabular}




\section{Comentario}

El tabaquismo es la principal causa de muerte evitable en el mundo. En Colombia mueren cada año alrededor de 20.000 personas por causas atribuibles al cigarrillo. No obstante, la encuesta nacional de salud de 2007 sugiere que se ha presentado una reducción de la prevalencia de tabaquismo en el país, con cifras reportadas de $12,8 \%$ y $21 \%(2,3)$.

Dentro de las intervenciones disponibles para dejar de fumar se incluyen consejería breve e intensiva, medicamentos y terapias alternativas.

Se ha demostrado que el uso de fármacos es la intervención que más aumenta las tasas de cesación (4); sin embargo, la disponibilidad de moléculas consideradas de primera línea, es limitada por falta de cubrimiento por el sistema de salud o por ausencia de información acerca de la efectividad y seguridad del medicamento (5).

Se han reportados estudios recientes con citisina, un medicamento emergente que previamente venía siendo utilizado en Europa del Este por más de 50 años para la cesación del tabaquismo. Citisina es un agonista parcial del receptor nicotínico, similar a la vareniclina pero a diferencia de ésta, que es sintética, es un derivado natural de la raíz del árbol de Cystisus laborinum y actualmente sólo tiene licencia para comercializarse en Europa del Este. Desde 2006, tras una revisión realizada por Etter, y conociendo su mecanismo de acción similar al de la vareniclina, se han realizado más estudios a fin de evaluar el riesgo, beneficio y costo de la terapia con citisina con miras a lograr su comercialización fuera de Europa del Este.

En lo concerniente a las intervenciones realizadas que han demostrado incrementos en la tasa de cesación, está la consejería, con tasas a seis meses que fluctúan entre 2,1 y $5,1 \%$ en ensayos clínicos (7). Un metaanálisis realizado por Fiore y colaboradores (6) evidencia que la consejería menor de tres minutos incrementa las tasas de abstinencia 1,3 veces, mientras que la consejería entre tres y diez minutos muestra un incremento de la tasa de 5,1 veces. De igual forma, las intervenciones intensivas demostraron mayor efectividad que la consejería breve con incremento de la tasa de abstinencia 6,1 veces.
En cuanto a los medicamentos estudiados, Fiore y colaboradores realizaron un metaanálisis basado en 80 estudios, en el que encontraron tasas de abstinencia a seis meses con uso de terapia con reemplazo de nicotina (TRN) entre 19,0\% (goma de mascar) y $26,7 \%$ (atomizador nasal) e incrementos del riesgo absoluto respecto al placebo entre 5,2\% y $12,9 \%$ respectivamente (OR 2,5 y 1,3 IC 95\% 1,2-1,7 y $1,7-3,0)(6)$. Ninguna forma de TRN ha demostrado superioridad sobre otra $(6,8)$.

De otra parte, un metaanálisis de Cochrane basado en 36 estudios, compara bupropión SR con placebo y muestra tasas de abstinencia a seis meses del $17 \%$ vs. 9\% respectivamente (RR 1,69 IC 95\% $1,53-1,85)(9)$, datos que fueron respaldados por $\mathrm{Wu}$ y Fiore (6).

La vareniclina también está en primera línea como lo respalda un metaanálisis de Fiore y colaboradores en el que se evidencian tasas de abstinencia comparada con placebo que demuestran que el uso de vareniclina $1 \mathrm{mg}$ al día, hace 33,2 veces más probable que el paciente abandone el tabaquismo comparado con el placebo, que lo hace sólo 13,8 veces (11). No obstante, con este medicamento hubo reporte de efectos neuropsiquiátricos y cardiovasculares.

Respecto a la terapia con citisina, se cuenta con un estudio publicado en el New England Journal of Medicine en 2011, en el que, al compararla con placebo, demostró tasas de abstinencia a 1 año de $8,4 \%$ vs. $2,4 \%$, con resultados estadísticamente significativos (IC95\% 2,3-9,2; p=0,001). Como desenlace secundario, la abstinencia sostenida a los 6 meses fue de $10 \%$ vs. 3,5\% comparado con placebo, con resultados estadísticamente significativos (IC $95 \% ; 2,9-10,1 ; \mathrm{p}<0,001)$. Se reportaron más efectos adversos en el grupo de citisina, con 1,7 veces más probabilidad de desarrollarlos (IC95\%, 1,4-2; $\mathrm{p}>0,001)$. Posteriormente, en 2014, se llevó a cabo un estudio de no inferioridad publicado en la misma revista, en el que se comparó citisina vs. nicotina y se demostraron tasas de abstinencia a un mes más altas en el grupo de citisina que en el de TRN. Los pacientes que recibieron terapia con citisina tienen 9,3 veces más probabilidad de abandonar el tabaquismo 
que aquellos que reciben TRN, con un número necesario a tratar de 11 para observar el efecto. En el desenlace secundario a una semana, 1, 2 y 6 meses, el desenlace secundario fue similar al primario excepto a los seis meses cuando la diferencia no fue estadísticamente significativa. Sólo el 5\% de los pacientes descontinuó la citisina. La mayoría de síntomas fueron gastrointestinales y trastornos del sueño, muy similares a los que se reportan con vareniclina. No se observaron efectos cardiovasculares ni psiquiátricos con vareniclina; no obstante, el estudio no tenía el tamaño suficiente para demostrarlos. Los síntomas anteriores no han sido reportados en Europa del Este; sin embargo, no se conoce el funcionamiento del sistema de reporte de eventos adversos allí.

A pesar de las dificultades descritas, la citisina parece ser una terapia efectiva para la cesación del tabaquismo si se considera que los estudios han demostrado efectividad, perfil de seguridad adecuado y bajo costo. De acuerdo con su mecanismo de acción similar al de la vareniclina, cabría diseñar un estudio para evaluar de forma apropiada el perfil de seguridad, la efectividad y los eventos adversos del medicamento en comparación con vareniclina, con el objetivo de plantear una nueva alternativa para el abandono del hábito tabáquico.

De cara a las limitaciones descritas, los resultados publicados resultan consecuentes, y por tanto se considera importante evaluar la posibilidad de realizar estudios en nuestra población para determinar la eficacia y seguridad del medicamento.

\section{Conflicto de intereses}

Alejandra Cañas Arboleda ha recibido patrocinio de Pfizer, AstraZeneca, Novartis, Glaxo y Bayer para la asistencia a congresos científicos y ha participado en estudios patrocinados por Glaxo y Sanofi.

Los demás autores no declaran conflictos de interés.

\section{Bibliografía}

1. Alba LH, Murillo R, Becerra N, Páez N, Cañas A, Mosquera C, et al. Recomendaciones para la cesación de la adicción al tabaco en
Colombia: guía de práctica clínica basada en la evidencia. Biomédica. 2013; 33(2) 186-204.

2. Ministerio de la Protección Social, Colciencias, CENDEX, S.E.I S.A. Encuesta Nacional de Salud 2007. $1^{\text {a }}$ Ed. Bogotá D.C.: Ministerio de la Protección Social; 2009.

3. Ministerio de Salud. II Estudio nacional de factores de riesgo de enfermedades crónicas- ENFREC II. Bogotá D.C: Ministerio de Salud; 1999.

4. República de Colombia. Ley 1335 de 2009. Diario Oficial No. 47.417 de 21 de julio de 2009.

5. Lemmens V, Oenema A, Knut IK, Bruj J. Effectiveness of smoking cessation intervention among adults: a systematic review of reviews. Eur J Cancer Preven. 2008;17:535-44. doi.org/10.1097/ CEJ.0b013e3282f75e48

6. Fiore MC, Jaén CR, Baker TB, Bailey WC. Treating tobacco use and dependence: 2008 update. Clinical practice guideline. Rockville, MD: U.S. Department of Health and Human Services. Public Health Service; 2008.

7. National Institute for Health and Clinical Excellence (NICE). Brief interventions and referral for smoking cessation in primary care and other. Marzo, 2006.

8. Ministry of Health. New Zealand smoking cessation guidelines. Wellington: Ministry of Health; 2007.

9. Hughes JR, Stead LF, Lancaster T. Antidepressants for smoking cessation. Cochrane Database Syst Rev. 2007;1:CD000031.http://dx.doi. org/10.1002/14651858.CD000031

10. Wu P, Wilson K, Dimoulas P, Mills EJ. Effectiveness of smoking cessation therapies: a systematic review and meta-analysis. BMC Public Health. 2006;6:300. doi.org/10.1186/1471-2458-6-300

11. Singh S, Loke YK, Spangler JG, Furberg CD. Risk of serious adverse cardiovascular events associated with varenicline: a systematic review and meta-analysis. CMAJ. 2011;183:1359-66. .doi.org/10.1503/ cmaj. 110218

12. Wagena EJ, Knipschild P, Zeegers MP. Should nortriptyline be used as a first-line aid to help smokers quit? Results from a systematic review and meta-analysis. Addiction. 2005;100:317-26. doi.org/10.1111/ j.1360-0443.2005.00998.x

13. Ministry of Health. New Zealand smoking cessation guidelines. Wellington: Ministry of Health; 2007.

14. Gourlay SG, Stead LF, Benowitz N. Clonidine for smoking cessation (Review). Cochrane Database Syst Rev. 2004;3:CD000058. doi. org/10.1002/14651858.CD000058.pub2

15. National Institute for Health and Clinical Excellence (NICE). Smoking cessation services in primary care, pharmacies, local authorities and workplaces, particularly for manual working groups, pregnant women and hard to reach communities. London: National Institute for Health and Clinical Excellence; 2008.

16. Etter JF. Cytisine for smoking cessation: a literature review and a metaanalysis. Arch Intern Med. 2006;166:1553-9.

17. Hajek $P$, McRobbie H, Myers K. Efficacy of cytisine in helping smokers quit: systematic review and meta-analysis. Thorax. 2013;68:1037-42.

18. Cahill K, Stead LF, Lancaster T. Nicotine receptor partial agonists for smoking cessation. Cochrane Database of Syst Rev. 2011;2:CD006103. 